\title{
Comparing nonlinear hydrodynamic forces in heaving point absorbers and oscillating wave surge converters
}

\author{
Giuseppe Giorgi $^{1}$ (I) $\cdot$ John V. Ringwood ${ }^{1}$
}

Received: 17 February 2017 / Accepted: 16 August 2017 / Published online: 29 August 2017

(C) Springer International Publishing AG 2017

\begin{abstract}
Two of the most common modes of oscillation of single degree of freedom wave energy converters are heave and surge, which are, respectively, exploited by heaving point absorbers (HPAs), and oscillating wave surge converters (OWSCs). Given major hydrodynamic differences between HPAs and OWSC, different nonlinear forces may be more or less relevant. Likewise, the scaling properties of such nonlinear forces may be different, according to the type of device, introducing uncertainties. This paper studies different nonlinear effects, and the relevance of different hydrodynamic force components, in HPAs and OWSCs. Nonlinear FroudeKrylov forces, as well as viscous drag effects, are represented and both prototype and full-scale device sizing are considered. Results show that HPAs are predominantly affected by nonlinear Froude-Krylov forces, while the most important hydrodynamic forces in OWSCs are diffraction and radiation effects. In addition, viscous drag appears to have little relevance in HPAs, but a significant influence in OWSCs. Finally, nonlinearities are shown to significantly affect the phase of the different force components.
\end{abstract}

Keywords Heaving point absorber - Oscillating wave surge converter · Nonlinear Froude-Krylov force · Viscous drag

Giuseppe Giorgi

giuseppe.giorgi.2015@mumail.ie

John V. Ringwood

john.ringwood@nuim.ie

1 Centre for Ocean Energy Research, Maynooth University, Maynooth, Kildare, Ireland

\section{Introduction}

Wave energy converters (WECs) are often classified based on the operating principles they use to absorb energy from ocean waves. Such operating principles refer to the modes in which the device oscillates, due to the action of the waves. Two of the most common modes are heave and surge, which are exploited, respectively, by heaving point absorbers (HPAs) and oscillating wave surge converters (OWSCs).

Due to different geometry, characteristic dimension, and degree of freedom (DoF), very different fluid-structure interaction mechanisms govern the motion of such devices; therefore, major hydrodynamic differences exist between HPAs and OWSCs (Folley et al. 2015). Nevertheless, the same linear model structure is commonly utilized, to define a mathematical model for the physical system. Unfortunately, the linearising assumption of small motion is critically challenged when a control strategy is included which, with the aim of increasing the power capture, magnifies the motion of the device, consequently increasing the relevance of nonlinearities, as shown by Giorgi et al. (2016b).

Given different hydrodynamic characteristics of HPAs and OWSCs, different nonlinear forces may be less or more relevant, depending on the device operating principle. Likewise, scaling properties of such nonlinear forces may be different according to the type of device. Scalability of nonlinear forces is particularly important when tests with prototypes are used to predict the performance of the device at full scale.

Candidates to incorporate significant nonlinear effects into the WEC model appear to be Froude-Krylov (FK) forces, and viscous drag forces, while the linear formulation of diffraction and radiation forces is normally assumed to be accurate enough, especially when the characteristic length of the device is much smaller than the wave length (Falnes 2002; Clement and Ferrant 1988). 
Fig. 1 Full-scale devices under study: On the left, a spherical heaving point absorber in deep water with radius $R$ of $2.5 \mathrm{~m}$, with the centre of gravity $G$ at the still water level (SWL); on the right, a prismatic oscillating wave surge converter, hinged at $4 \mathrm{~m}$ from the sea bottom, and piercing $3 \mathrm{~m}$ from the SWL when at rest. Both devices have a power take-off (PTO) system with a damping and a stiffness term. The PTO representation for the OWSC is simply conceptual, since the actual PTO operates on the rotational quantities of $\delta$ and $\dot{\delta}$
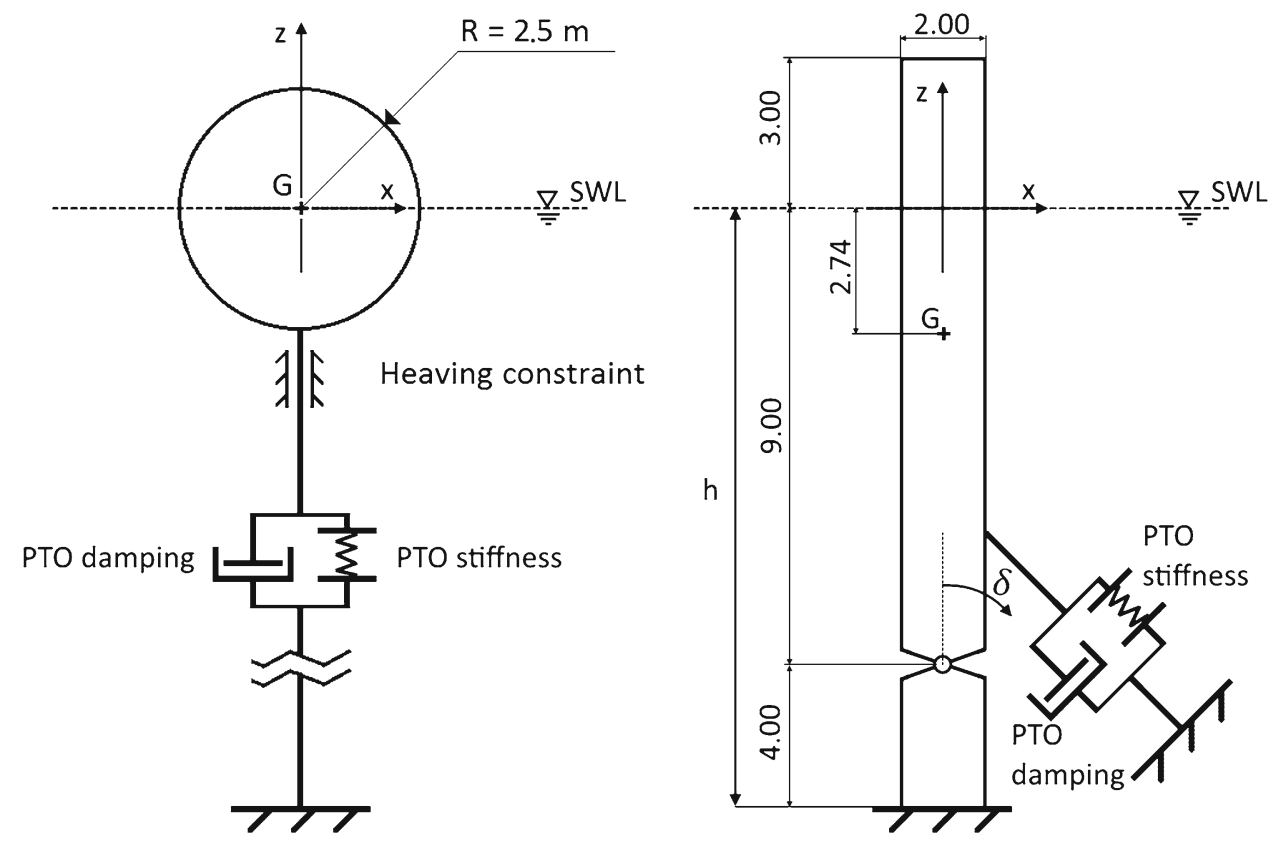

In the literature, different approaches can be found for modelling nonlinear FK forces for HPAs (Gilloteaux 2007; Giorgi and Ringwood 2016), and OWSCs (Giorgi et al. 2016a). Viscous drag forces are usually included in the model by means of a Morison-like term (Lok et al. 2014; Bhinder et al. 2012). It is worth mentioning that a full validation of the nonlinear models proposed in this paper is not yet complete. Despite the fact that some validation of the methods applied to describe nonlinear FK and drag forces can be found in the literature, to the best of knowledge of the authors, no previous work has used both nonlinear FK and drag force under similar controlled conditions. Gilloteaux (2007) validates nonlinear FK forces for a point absorber using the same method as in this paper, but without including viscous drag; viscous drag force using the Morison equation has been validated for a HPA (Bhinder et al. 2011) and OWSC (Bhinder et al. 2015), but using a fixed body or a free decay experiment, therefore without a controller exaggerating the amplitude of motion. Finally, a first validation of the nonlinear Froude-Krylov and drag forces under control for HPA using a numerical wave tank has been proposed in Giorgi and Ringwood (2017).

This paper attempts to develop parsimonious hydrodynamic models for HPAs and OWSCs by combining, as appropriate, linear and nonlinear hydrodynamic forces in a boundary element formulation and considering both prototype and full-scale models. Indeed, including nonlinearities may increase the model accuracy, but at an additional computational burden. A model is parsimonious if describes only relevant nonlinearities, in the attempt to realize the best compromise between model fidelity and computational cost. Crucially, parsimonious dictates that OWSC and HPA models differ in model structure.
The reminder of the paper is organized as follows: Sect. 2 presents the nonlinear hydrodynamic models, which are applied to a case study, presented in Sect. 3. Results are given in Sect. 4, and some conclusions and final remarks are presented in Sect. 5.

\section{Hydrodynamic models}

This paper considers two single-DoF devices, as shown in Fig. 1: a heaving point absorber, which is constrained to translate in the vertical direction only, and an oscillating wave surge converter, which pitches around a hinge, with angle $\delta$. More detailed illustrations of the HPA and the OWSC are shown in Figs. 2 and 3, respectively. The dimensions shown in Fig. 1 refer to a likely full-scale size.

Under the assumption of inviscid fluid, and irrotational and incompressible incident flow, Newton's second law of dynamics can be applied (Merigaud et al. 2012), so that, for the HPA:

$m \ddot{z}(t)=F_{\mathrm{g}}-\iint_{S(t)} P(t) \mathbf{n} \mathrm{d} S+F_{\mathrm{PTO}}(t)$,

while, for the OWSC:

$I \ddot{\delta}(t)=F_{\mathrm{g}} \times L_{\mathrm{g}}-\iint_{S(t)} P(t) \mathbf{n} \times l \mathrm{~d} S+T_{\mathrm{PTO}}(t)$,

where $m$ and $I$ are, respectively, the mass of the HPA and the pitching inertia of the OWSC, $F_{\mathrm{g}}$ is the gravity force, $L_{\mathrm{g}}$ the distance between the centre of gravity of the OWSC and the hinge, $S$ the submerged surface, $P$ the pressure of the fluid on 
Fig. 2 Axisymmetric heaving device with generic profile $f(\sigma)$ : the figure on the left shows the rest position, with the centre of gravity $G$ at the still water level (SWL) and draft $h_{0}$; the figure on the right shows the free surface elevation $\eta$ and the device displacement $z_{\mathrm{d}}$ after a time $t^{*}$. The pressure is integrated over the surface between $\sigma_{1}$ and $\sigma_{2}$ (Giorgi and Ringwood 2016)

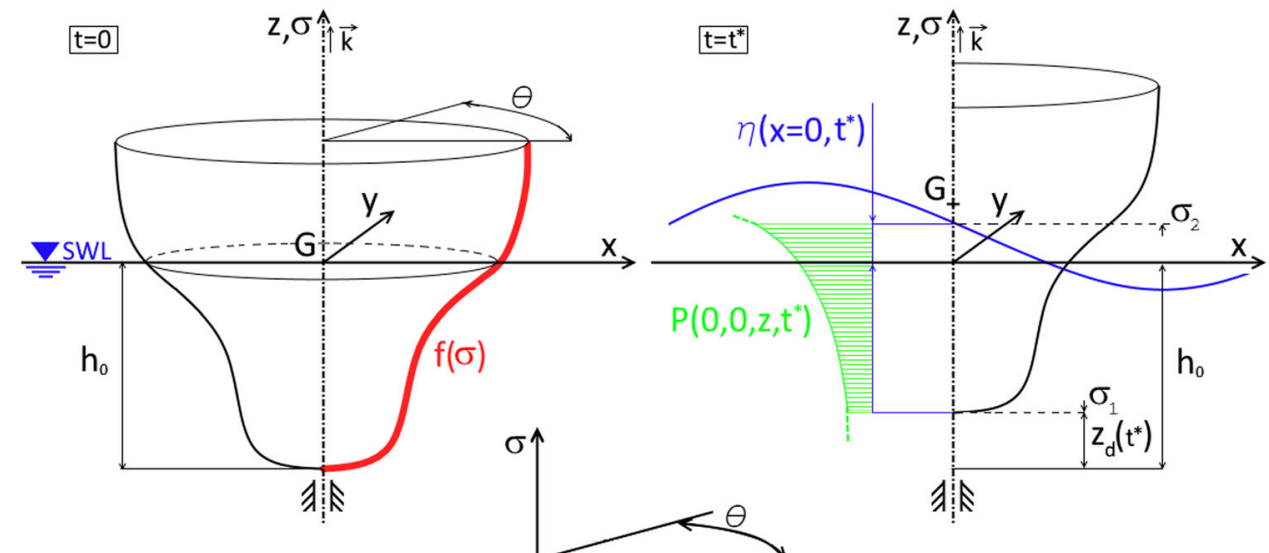

(a)

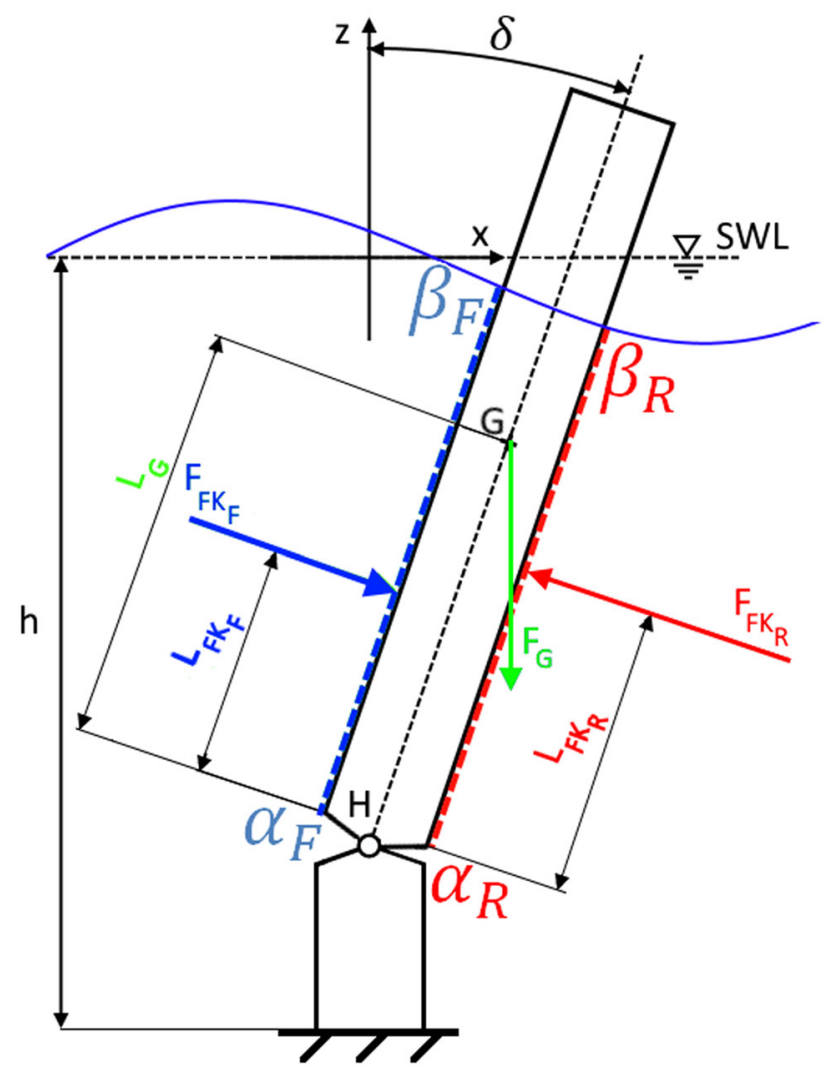

Fig. 3 Scheme for nonlinear Froude-Krylov force calculation for an oscillating wave surge converter. The static and dynamic pressures act on the front and rear surfaces of the flap, delimited by $\alpha_{\mathrm{F}}$ and $\alpha_{\mathrm{R}}$ at the bottom, and $\beta_{\mathrm{F}}$ and $\beta_{\mathrm{R}}$ at the intersection between the free surface and the body

the body surface, and $\mathbf{n}$ the vector normal to the infinitesimal surface $\mathrm{d} S$, which is a distance $l$ from the hinge in the case of the OWSC. Finally, the power take-off system applies a force $\left(F_{\mathrm{PTO}}\right)$ on the HPA and a torque $\left(T_{\mathrm{PTO}}\right)$ on the OWSC.

According to Bernoulli's equation (Falnes 2002), the fluid pressure $P$ acting of the surface of the structure is obtained as:

$$
P(t)=-\rho g z(t)-\rho \frac{\partial \phi(t)}{\partial t}-\rho \frac{|\nabla \phi(t)|^{2}}{2}
$$

where $\rho$ is the density of the water, $g$ the acceleration of gravity, $-\rho g z$ the hydrostatic pressure $\left(P_{\mathrm{st}}\right)$, and $\phi$ the potential flow which, based on linear wave theory, is the sum of the undisturbed incident flow potential $\phi_{\mathrm{I}}$, the diffraction potential $\phi_{\mathrm{D}}$, and the radiation potential $\phi_{\mathrm{R}}$ :

$\phi(t)=\phi_{\mathrm{I}}(t)+\phi_{\mathrm{D}}(t)+\phi_{\mathrm{R}}(t)$

Omitting the time-dependence annotation for brevity, substituting Eqs. (3) and (4) into (1) and (2), and adding a viscous drag term, one obtains, for the HPA:

$m \ddot{z}=F_{\mathrm{FK}}+F_{\mathrm{D}}+F_{\mathrm{R}}+F_{\mathrm{vis}}+F_{\mathrm{PTO}}$,

while, for the OWSC:

$I \ddot{\delta}=T_{\mathrm{FK}}+T_{\mathrm{D}}+T_{\mathrm{R}}+F_{\mathrm{vis}}+T_{\mathrm{PTO}}$,

where $F$ and $T$ are the force and torque applied on the HPA and OWSC, respectively, and the subscripts FK, D, R, vis, and PTO refer to FK, diffraction, radiation, viscous drag, and PTO, respectively. Note that the FK force is composed of a static and a dynamic part; furthermore, the sum of the dynamic FK and diffraction forces is usually referred to as the excitation force. The detailed definition and implementation of each component of (5) and (6) is given in Sects. 2.12.4. As a common assumption (Falnes 2002), the nonlinear quadratic terms in (3) are neglected, and only linear potentials are considered, since the vast majority of waves in the power production region are appropriately represented by linear wave theory.

\subsection{Diffraction term}

The diffraction force/torque is the integral over the wetted surface of the diffraction pressure $P_{\mathrm{D}}=-\rho \frac{\partial \phi_{\mathrm{D}}}{\partial t}$. Assum- 
ing only small wave amplitude and steepness, the potential problem is linearized and solved around the equilibrium position of the device. Therefore, the mean wetted surface $S_{\mathrm{M}}$ is considered, and the diffraction term is computed through the convolution product between the diffraction impulse response function (IRF) $K_{\mathrm{D}}$ and the free surface elevation $\eta$. The resulting radiation force for the HPA is

$$
F_{\mathrm{D}}=-\iint_{S_{M}} P_{\mathrm{D}} \mathbf{n} \mathrm{d} S=-\int_{-\infty}^{\infty} K_{\mathrm{D}}^{\mathrm{HPA}}(t-\tau) \eta(\tau) \mathrm{d} \tau,
$$

while the radiation torque for the OWSC is

$$
T_{\mathrm{D}}=-\iint_{S_{M}} P_{\mathrm{D}} \mathbf{n} \times l \mathrm{~d} S=-\int_{-\infty}^{\infty} K_{\mathrm{D}}^{\mathrm{OWSC}}(t-\tau) \eta(\tau) \mathrm{d} \tau
$$

The impulse response functions for each device are obtained from the boundary element method (BEM) software WAMIT (WAMIT Inc. 2013).

\subsection{Radiation term}

The radiation force/torque is the integral over the wetted surface of the radiation pressure $P_{\mathrm{R}}=-\rho \frac{\partial \phi_{\mathrm{R}}}{\partial t}$. As in Sect. 2.1, the radiation term is computed under linear assumptions. Using Cummins equation (Cummins 1962), the radiation term is computed, for the HPA, as

$F_{\mathrm{R}}=-m_{\infty} \ddot{z}-\int_{-\infty}^{\infty} K_{\mathrm{R}}^{\mathrm{HPA}}(t-\tau) \dot{z}(\tau) \mathrm{d} \tau$

and for the OWSC, as

$T_{\mathrm{R}}=-I_{\infty} \ddot{\delta}-\int_{-\infty}^{\infty} K_{\mathrm{R}}^{\mathrm{OWSC}}(t-\tau) \dot{\delta}(\tau) \mathrm{d} \tau$,

where $m_{\infty}$ and $I_{\infty}$ are, respectively, the added mass at infinite frequency of the HPA, and the added inertia at infinite frequency of the OWSC, and $K_{\mathrm{R}}$ is the radiation impulse response function.

The computationally expensive radiation convolution product is replaced by its state space representation, as shown by Taghipour et al. (2007).

\subsection{Froude-Krylov term}

The FK force is divided into two parts, static and dynamic, where the static FK force is the balance between the gravity force, and the integral over the wetted surface of the static pressure $P_{\text {st }}$, while the dynamic FK force refers to the integral over the wetted surface of the dynamic pressure, derived from the incident field potential as $P_{\mathrm{dy}}=-\rho \frac{\partial \phi_{I}}{\partial t}$. The integral is nonlinearly computed over the instantaneous wetted surface of the devices, considering the instantaneous intersection between the body and the free surface elevation. Due to the different shape and mode of oscillation of HPAs and OWSCs, the formulation of the nonlinear FK is analyzed separately.

\subsubsection{Froude-Krylov force for heaving point absorbers}

Applying Airy's wave theory in deep water conditions, and assuming the origin of the frame of reference to be at the still water level (SWL), the total pressure is obtained as follows:

$P(x, z, t)=P_{\mathrm{st}}+P_{\mathrm{dy}}=-\rho g z+\rho g a \mathrm{e}^{\chi z} \cos (\omega t-\chi x)$

where $x$ is the direction of wave propagation, $a$ is the wave amplitude, $\chi$ the wave number and $\omega$ the wave frequency.

As shown in detail in Giorgi and Ringwood (2016), an algebraic solution is achievable for any axisymmetric heaving point absorber, in deep water conditions, such as the one in Fig. 2, which can be described by parametric cylindrical coordinates $[\sigma, \theta]$ as:

$\left\{\begin{array}{l}x(\sigma, \theta)=f(\sigma) \cos \theta \\ y(\sigma, \theta)=f(\sigma) \sin \theta \quad, \quad \sigma \in\left[\sigma_{1}, \sigma_{2}\right] \wedge \theta \in[0,2 \pi) \\ z(\sigma, \theta)=\sigma\end{array}\right.$

Referring to the notation in Fig. 2, the resulting FK heave force is computed as follows:

$F_{\mathrm{FK}_{z}}=F_{g}-\int_{0}^{2 \pi} \int_{\sigma_{1}}^{\sigma_{2}} P(x(\sigma, \theta), z(\sigma, \theta), t) f^{\prime}(\sigma) f(\sigma) \mathrm{d} \sigma \mathrm{d} \theta$

where the subscript $z$ indicates the heave component of the FK force; the limits of integration, defining the instantaneous wetted surface, are $\sigma_{1}=z_{\mathrm{d}}-h_{0}$ and $\sigma_{2}=\eta$.

The algebraic solution of (13) is straightforward and easily implemented in the simulation model.

\subsubsection{Froude-Krylov torque for oscillating wave surge converters}

Since the OWSC is a bottom-hinged device, the pressure formulation in intermediate depth water conditions needs to be used:

$$
P(x, z, t)=-\rho g z+\rho g a \frac{\cosh (\chi(z+h))}{\cosh (\chi h)} \cos (\omega t-\chi x)
$$


where $h$ is the water depth.

The static and dynamic pressures simultaneously act perpendicularly on the front and rear surfaces of the OWSC, which have opposite normals, generating, as a consequence, opposite torques. The torque due to the pressure on each side of the OWSC is computed as the outer product between the resulting force $F_{\mathrm{FK}}$ and its distance $L_{\mathrm{FK}}$ from the hinge:

$$
\begin{aligned}
F_{\mathrm{FK}} & =-\int_{-\frac{W}{2}}^{\frac{W}{2}} \int_{\alpha_{\mathrm{F}}}^{\beta_{\mathrm{F}}} P \mathbf{n} \frac{\mathrm{d} x \mathrm{~d} y}{\sin \delta} \\
L_{\mathrm{FK}_{\mathrm{F}}} & =\frac{1}{F_{\mathrm{FK}}} \int_{-\frac{W}{2}}^{\frac{W}{2}} \int_{\alpha_{\mathrm{F}}}^{\beta_{\mathrm{F}}} P \mathbf{n} \times l \frac{\mathrm{d} x \mathrm{~d} y}{\sin \delta} \\
F_{\mathrm{FK}_{\mathrm{R}}} & =-\int_{-\frac{W}{2}}^{\frac{W}{2}} \int_{\alpha_{\mathrm{R}}}^{\beta_{\mathrm{R}}} P \mathbf{n} \frac{\mathrm{d} x \mathrm{~d} y}{\sin \delta} \\
L_{\mathrm{FK}_{\mathrm{R}}} & =\frac{1}{F_{\mathrm{FK}}} \int_{-\frac{W}{2}}^{\frac{W}{2}} \int_{\alpha_{\mathrm{R}}}^{\beta_{\mathrm{R}}} P \mathbf{n} \times l \frac{\mathrm{d} x \mathrm{~d} y}{\sin \delta}
\end{aligned}
$$

where $F$ and $R$ subscripts refer to the front and rear surface, respectively, and the infinitesimal area of the flap rotated by an angle $\delta$ is $\mathrm{d} S=\frac{\mathrm{d} x \mathrm{~d} y}{\sin \delta}$. The limits of integration are defined by $W$, which is the width of the flap along the $y$ axis, and by $\alpha_{\mathrm{F}}, \alpha_{\mathrm{R}}, \beta_{\mathrm{F}}$ and $\beta_{\mathrm{R}}$, which define the surface on which the pressure is acting, as shown in Fig. 3. If the body is piercing the water, $\beta_{\mathrm{F}}$ and $\beta_{\mathrm{R}}$ are the submerged lengths of the flap; if the device becomes fully submerged (when large motions occur), $\beta_{\mathrm{F}}$ and $\beta_{\mathrm{R}}$ are the extremities of the flap.

The total FK torque is the sum of the torque due to gravity, and the torques due to the static and dynamic pressures on the front and rear surfaces:

$T_{\mathrm{FK}}=F_{\mathrm{g}} \times L_{\mathrm{g}}+F_{\mathrm{FK}_{\mathrm{F}}} \times L_{\mathrm{FK}_{\mathrm{F}}}+F_{\mathrm{FK}_{\mathrm{R}}} \times L_{\mathrm{FK}_{\mathrm{R}}}$

\subsection{Viscous drag term}

\subsubsection{Viscous drag force for heaving point absorbers}

The viscous drag force calculation is based on the Morison equation (Morison et al. 1950), which takes into account the shape of the device vie the drag coefficient $C_{\mathrm{d}}$, the size of the floater with the characteristic area $A_{\mathrm{d}}$, and the relative velocity between the velocity of the floater $V$ and the vertical component $V_{0_{z}}$ of the undisturbed flow velocity (Babarit et al. 2012):

$$
F_{\text {vis }}=-\frac{1}{2} \rho C_{\mathrm{d}} A_{\mathrm{d}}\left|V-V_{0_{z}}\right|\left(V-V_{0_{z}}\right)
$$

Note that the characteristic surface area $A_{\mathrm{d}}$ is the projection of the instantaneous wetted surface onto the plane normal to the flow. Therefore, as the the instantaneous wet- ted surface is considered, $A_{\mathrm{d}}$ changes as the device pierces the water during its motion.

The value of the drag coefficient $C_{\mathrm{d}}$ can be estimated for simple geometries by the Keulegan-Carpenter number $(K C)$, which is a dimensionless quantity defined as the ratio between drag and inertia forces, acting on a body in an oscillatory fluid flow (Keulegan and Carpenter 1956). In case of sinusoidal motion, the $K C$ number can be computed as:

$K C=2 \pi \frac{A}{L_{\mathrm{c}}}$

where $A$ is the amplitude of motion and $L_{\mathrm{c}}$ is the characteristic length scale, which is equal to the diameter, in the case of a sphere. The motion of the HPA and the diameter of the floater are of the same order of magnitude, which means that the $K C$ number is about $2 \pi$ so, according to Molin (2002), $C_{\mathrm{d}}$ can be taken equal to 1 . However, it is worth pointing out that such a method for estimating the drag coefficient is subject to some uncertainty, which could be assessed through a sensitivity analysis, as shown by Babarit et al. (2012).

\subsubsection{Viscous drag torque for oscillating wave surge converters}

As in Sect. 2.4.1, the drag torque is computed by applying the Morison equation (Morison et al. 1950). However, since the device is rotating, the relative linear velocity between the body and the fluid is progressively changing, while moving away from the hinge. Furthermore, as intermediate depth water conditions are used, the fluid velocity is significantly changing with depth, due to the proximity of the bottom. Therefore, the wetted surface of the flap is equally divided into $N_{\mathrm{s}}=10$ horizontal sections, and the total viscous torque $T_{\text {vis }}$ is computed as follows (Babarit et al. 2012):

$T_{\mathrm{vis}}=\sum_{i=1}^{N_{\mathrm{s}}=10} L_{i} \times\left(-\frac{1}{2} \rho C_{\mathrm{d}} A_{\mathrm{d}_{i}}\left|V_{i}-V_{0_{i}}\right|\left(V_{i}-V_{0_{i}}\right)\right)$

where $L_{i}$ is the distance from the centre of the $i$ th slice to the hinge, and $V_{i}=L_{i} \dot{\delta}$ is its velocity. In Sect. 2.4.1, the instantaneous wetted surface is similarly taken into account.

The number of sections $N_{\mathrm{s}}$ has been chosen as 10 to have a reasonable balance between computational time and accuracy. On the one hand, the higher the number of divisions, the smaller the width of each section; the smaller the variation of the velocities with respect to the centre of the section, the higher the accuracy of the computation. On the other hand, the computational time is directly proportional to the number of sections. 
The amplitude of motion of the top of the flap is likely to be of the same order of magnitude as the wave amplitude, which results in a Keulegan-Carpenter number less than one. According to Bearman et al. (1985), the drag coefficient for a plate in oscillatory flow at low $K C$ is $C_{\mathrm{d}}=7.8 K C^{-\frac{1}{3}}$, so $C_{\mathrm{d}}$ has been taken as 8. As already mentioned in Sect. 2.4.1, the estimation of the value of $C_{\mathrm{d}}$ is subject to some uncertainty (Babarit et al. 2012).

\section{Case study}

Figure 1 shows the geometries and full-scale dimensions of the HPA and the OWSC, which have been inspired, respectively, by the Wavestar HPA (Wavestar 2016) and the Oyster 2 OWSC (Aquamarine 2016). One of the inherent differences between HPAs and OWSCs is that OWSCs must be installed near shore, whereas HPAs may be installed either near or off-shore. Therefore, to highlight such a difference, related to the operating principle, deep water conditions are chosen for the HPA.

The HPA is a sphere of radius $R 2.5 \mathrm{~m}$, with the geometric and gravity centre at the SWL, designed to work in deep water conditions, constrained to heave $(z)$ and tethered to the seabed through a PTO mechanism, composed of stiffness and damping terms.

The OWSC is a rectangular prism, with dimensions according to the section shown in Fig. 1 and width $W$ of $26 \mathrm{~m}$. The position of the centre of gravity $G(2.74 \mathrm{~m}$ below the SWL), the mass $(150,000 \mathrm{~kg})$, and the inertia around the hinge $\left(8.12 \times 10^{6} \mathrm{~kg} \mathrm{~m}^{2}\right)$, as well as all the dimensions, have been taken from (Babarit et al. 2012). The flap rotates around the hinge with angle $\delta$, and a PTO with rotational stiffness and damping terms is implemented. Note that the PTO representation in Fig. 1 is simply conceptual, since the actual PTO operates on the rotational quantities $\delta$ and $\dot{\delta}$.

Incident regular waves are used, to study the hydrodynamic forces at each individual frequency. Wave periods $T_{\mathrm{w}}$ range from 5 to $15 \mathrm{~s}$, with a $1 \mathrm{~s}$ step, while wave heights $H_{\mathrm{w}}$ range from 0.5 to $3 \mathrm{~m}$, with a $0.5 \mathrm{~m}$ step. Note that some of the considered wave conditions (at large $H_{\mathrm{w}}$ and small $T_{\mathrm{w}}$ ) have a steepness too large to be appropriately described by linear Airy's theory, as assumed in Sect. 2. However, the objective of the paper is to focus on the comparative wave-structure interactions due to different WEC operating principles, which is facilitated by the use of identical input wave conditions.

The response of the device is analyzed under controlled conditions, since the action of the controller enlarges the amplitude of motion and increases the relevance of nonlinearities. In order to facilitate as close a comparison as possible, the controller structure has been chosen to be the same for both the HPA and the OWSC. Therefore, reactive control is applied, which tunes the stiffness and damping parameters of the PTO, $K_{\mathrm{PTO}}$ and $B_{\mathrm{PTO}}$, respectively, to maximize the power absorption for each wave condition. No constraints are imposed in the power optimization strategy, so that the $K_{\mathrm{PTO}}$ may take negative values, for example in the case where the device resonant frequency is less than the predominant wave frequency. Furthermore, no constrained are imposed on either device displacement, velocity, or PTO force. Simulations show that reactive control is effective for both the HPA and the OWSC, as it achieves the objective of increasing the power absorption compared to the case where the PTO acts just as a linear damper (uncontrolled condition).

The prototype model dimensions have been obtained by applying Froude similarity since, in wave energy applications, gravity and inertial forces are dominant (Heller 2011). According to Froude similarity, the dimensionless Froude number $(F r)$, which is the square root of the ratio between inertial and gravity forces, is kept constant. The scaling ratio $s$ has been chosen equal to $1: 20$ for both the HPA and OWSC, consistent with the prototype scale of the Wavestar (Hansen 2013), and Oyster devices (Whittaker et al. 2007).

The PTO coefficients, $K_{\mathrm{PTO}}$ and $B_{\mathrm{PTO}}$, have been chosen in order to optimize the power absorption of the full-scale device. These control parameters have been scaled down for the prototype model, according to Froude similarity. Note that, due to nonlinearities, such PTO parameters may be suboptimal for the small-scale device. Nevertheless, in real applications, the objective is to maximize the power absorption of the full-scale device, rather than the small-scale one. Furthermore, Froude-scaling the PTO coefficients facilitates the comparison between small and full-scale models since, with scaled PTO parameters, differences in the response (displacement, velocity, and forces) are due to nonlinearities only.

Likewise, the same drag coefficient has been used for the small and full-scale models. In general, $C_{\mathrm{d}}$ depends on the type of flow around the device; therefore, it is influenced by the Reynolds number ( $R e)$, which is the dimensionless number defined as the ratio between inertial and viscous forces. Froude-scaling ensures the same $\mathrm{Fr}$ number, implying a different $R e$ number instead. Nevertheless, it is assumed that the main contribution to drag forces is flow separation, associated with the inertia of the flow field, which is largely preserved under Froude similarity. Consequently, retaining the same $C_{\mathrm{d}}$ for small and full-scale devices is considered as a good first approximation. However, as for the PTO parameters, having the same drag coefficient makes the comparison more even, since differences arise from the model structure only, rather than from differently scaled coefficients. 


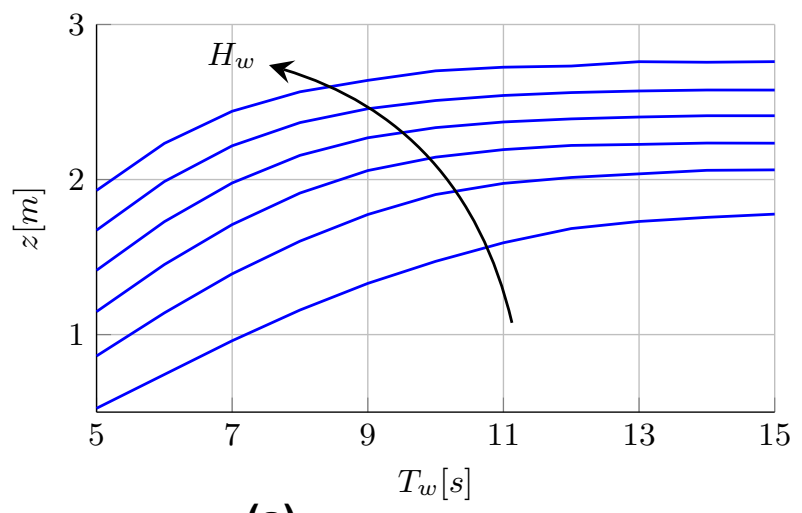

(a) Heaving point absorber.

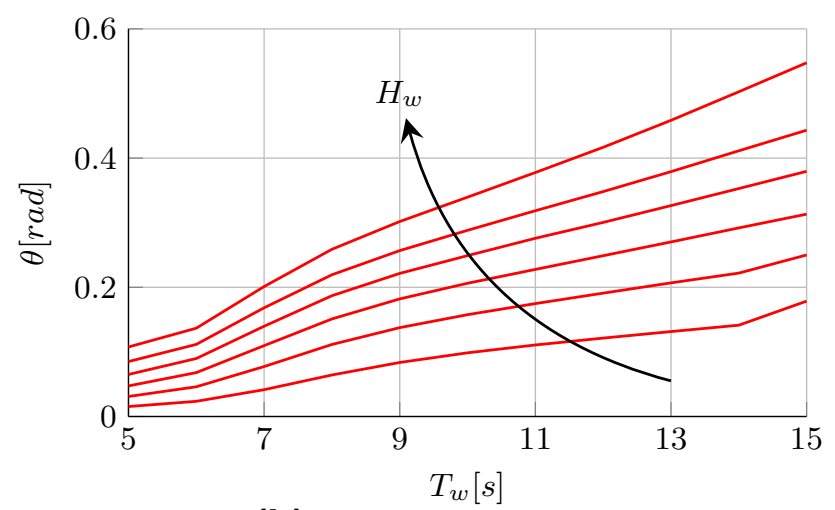

(b) Oscillating wave surge converter.

Fig. 4 Maxima of the steady state response for the heaving point absorber (a), and the oscillating wave surge converter (b)

\section{Results}

The responses of the HPA and OWSC, at full scale, are shown in Fig. 4, which presents the maxima of the vertical/angular displacements, computed once the transient has elapsed.

The main differences between the HPA and the OWSC arise from the comparative relevance of each of the hydrodynamic forces in each of the devices. Figure $5 \mathrm{a}, \mathrm{b}$ show the amplitude of the static FK $\left(\mathrm{FK}_{\mathrm{st}}\right)$, dynamic FK $\left(\mathrm{FK}_{\mathrm{dy}}\right)$, diffraction $(D)$, radiation $(R)$, and viscous drag (vis) forces and torques under controlled conditions, for the HPA and the OWSC, respectively, using a regular wave of $T_{\mathrm{w}}=10 \mathrm{~s}$ and $H_{\mathrm{w}}=1.5 \mathrm{~m}$. Due to nonlinearities, forces may be, in general, asymmetric; therefore, the force amplitude has been defined as half of the variation from peak to trough.

Finally, both model scales have been plotted together in Fig. 5: the results obtained with the prototype model have been scaled up, with a factor $s^{3}$ for the forces of the HPA, and $s^{4}$ for the torques of the OWSC. Forces and torques obtained with the prototype scale are identified by the subscript $p$, whereas $f$ indicates the full-scale model.

Overall, the nonlinear static FK term, which is the restoring force that pulls the device back into the equilibrium

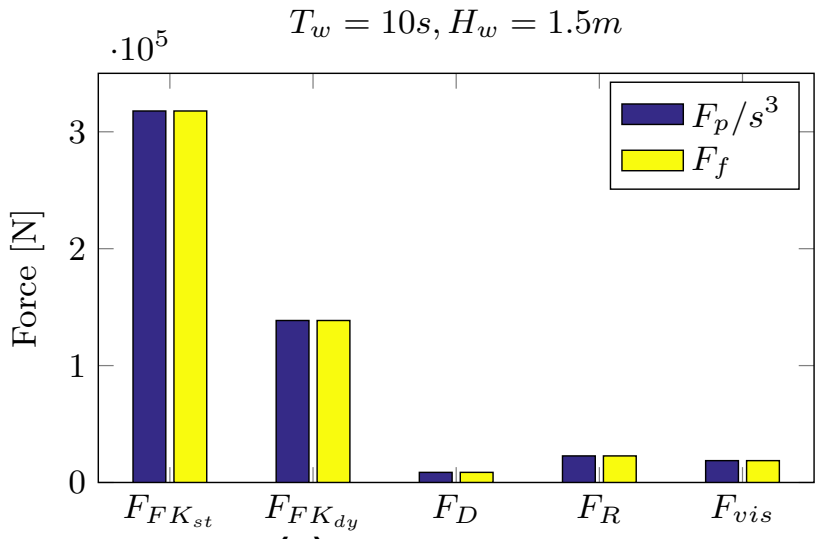

(a) Heaving point absorber.

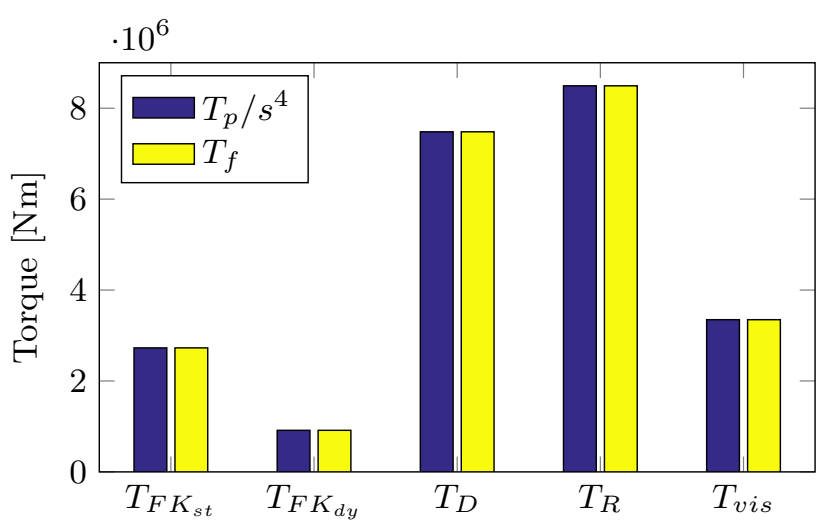

(b) Oscillating wave surge converter.

Fig. 5 Full-scale total hydrodynamic force $(F)$ and torque $(T)$ decomposition into static $\mathrm{FK}\left(\mathrm{FK}_{\mathrm{st}}\right)$, dynamic $\mathrm{FK}\left(\mathrm{FK}_{\mathrm{dy}}\right)$, diffraction $(D)$, radiation $(R)$, and viscous drag (vis), according to the prototype $(p)$ and full-scale $(f)$ model, using a regular wave of period $T_{\mathrm{W}} 10 \mathrm{~s}$ and height $H_{\mathrm{w}} 1.5 \mathrm{~m}$

position, is by far the largest component in HPAs. While the dynamics of the HPA are dominated by static and dynamic FK forces, in the OWSC the major hydrodynamic action is due to diffraction and radiation forces.

The components related to wave excitation are the dynamic FK force and the diffraction force. Figure 5 shows that HPAs are mainly excited by the dynamic FK force while, in OWSCs, diffraction forces are the most important wave excitation mechanism.

Radiation damping and viscous drag are the dissipative terms of the hydrodynamic force, with a notional linear and quadratic dependance on the velocity of the device, respectively. While in HPAs, dissipation terms are very small compared to the FK components, in OWSCs radiation and viscous drag are predominant.

Comparing small- and full-scale models, it is clear that all forces and torques are perfectly scalable, meaning that nonlinear FK and viscous drag models are invariant to scaling, for both the HPA and the OWSC. Consequently, as far as 
the nonlinear model structure is concerned, it is equivalent to consider small or full scale, as long as the same model parameters are chosen. However, even though the nonlinear model structure is scale independent, the values of the model parameters, in particular the viscous drag coefficient, may vary between different scales. Indeed, Clabby (2014) shows that the power captured during experimental tests for an OWSC at prototype scale is $7 \%$ greater, relative to full scale. Figure 5 suggests that such a difference is not due to the inherent structure of nonlinear forces, but may be attributed to different flow conditions at different scales, resulting in different values of the drag coefficient.

Following the scalability considerations, only the fullscale devices will be discussed hereafter.

Differences between the hydrodynamic forces, experienced by the HPA and the OWSC, are not only due to their amplitudes, as shown in Fig. 5, but also on the phase with which they are acting on the body as well. Notwithstanding the clear notion of phase between quasi-harmonic signals, a rigorous definition of phase is available only for sinusoidal signals. Nevertheless, a predominant phase $\varphi$ can be defined, using the delay at which the cross-correlation function has its maximum. Such phases are computed for all the forces $F$ (and torques $T$ ), with respect to the free surface elevation $\eta$, as follows:

$\varphi=\frac{2 \pi}{\underset{\tau}{\operatorname{argmax}}\left\{\int_{-\infty}^{\infty} F^{*}(t) \eta(t+\tau) \mathrm{d} t\right\}}$,

where $F^{*}$ denotes the complex conjugate of the force (or torque), and $\tau$ the time lag.

For all the hydrodynamic forces, such a phase difference is used to construct a phasor, whose length is equal to the exponential of the ratio between the amplitude of the force and the total hydrodynamic force. The phasor graphs for the HPA and the OWSC are shown in Figs. 6 and 7, respectively. The reason for scaling the length of the phasor with an exponential is to decrease the differences between the amplitudes of the different forces, facilitating comparative plotting.

To highlight the effect of nonlinearities, Figs. 6 and 7 are drawn for waves with the same period $T_{\mathrm{w}}=10 \mathrm{~s}$, and 6 heights $H_{\mathrm{w}}$ from 0.5 to $3 \mathrm{~m}$. Indeed, if the model was fully linear, the phase of each force would depend only on the wave frequency, while it would be independent of the wave height. On the contrary, nonlinear forces show a phase drift causing, in turn, a phase shift in position and velocity. Therefore, due to nonlinearities, as the wave height increases, the phasors are rotating in the direction indicated by the curved arrows in Figs. 6 and 7.

In fact, the only phasor that is not rotating is the diffraction one, which is linear, and does not depend on the response of the device. On the other hand, notwithstanding that the radiation force is linear, the radiation phasor is rotating, since it

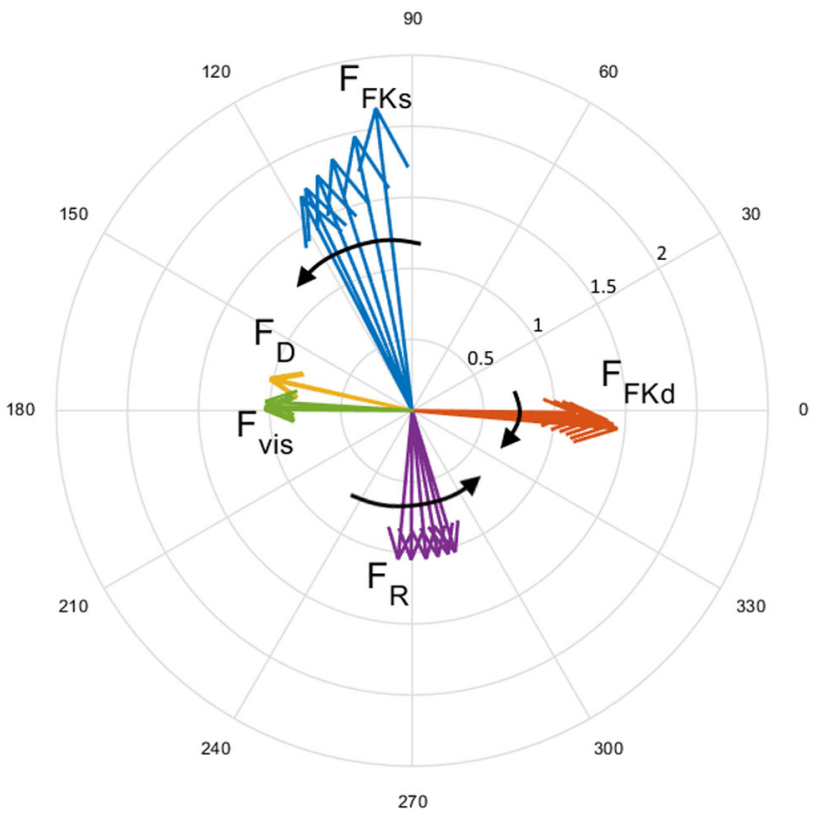

Fig. 6 Phasor graph for the HPA, for a wave period $T_{\mathrm{w}}=10 \mathrm{~s}$, and 6 heights $H_{\mathrm{w}}$ from 0.5 to $3 \mathrm{~m}$, increasing in the direction of the curved arrows

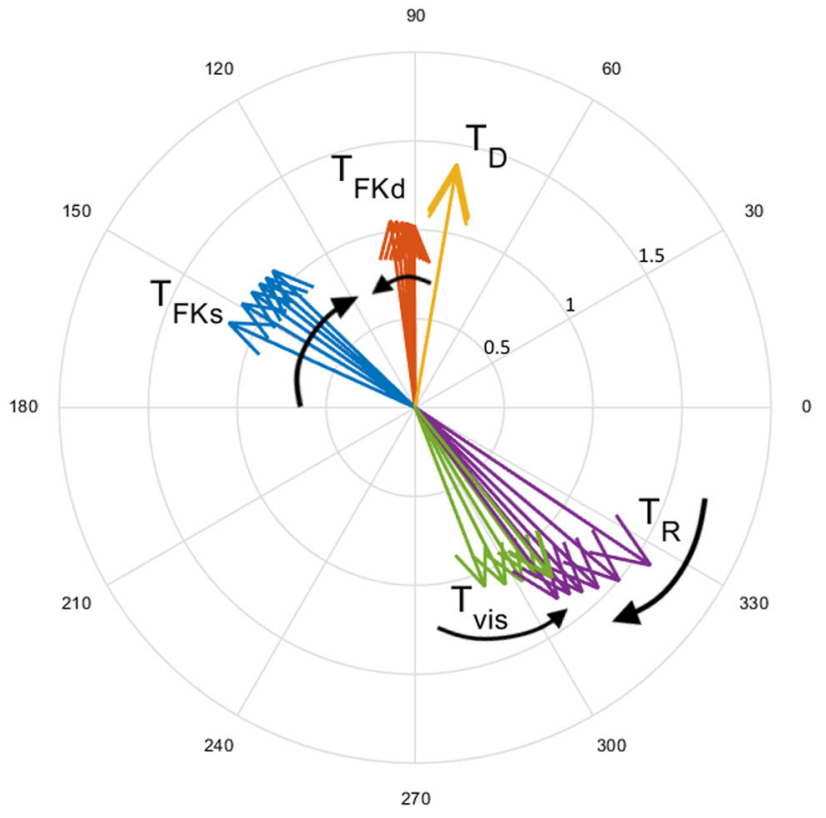

Fig. 7 Phasor graph for the OWSC, for a wave period $T_{\mathrm{w}}=10 \mathrm{~s}$, and 6 heights $H_{\mathrm{w}}$ from 0.5 to $3 \mathrm{~m}$, increasing in the direction of the curved arrows

depends on the velocity. Finally, FK and viscous drag phasors are rotating both due to their nonlinear nature, and their dependence on the displacement and velocity.

Nevertheless, the rotation of the dynamic FK phasor is relatively small, since it depends mainly on the free surface elevation. Conversely, static FK and radiation phasors rotate in the same direction, due to the large dependence on position 
Table 1 Phase range of the phasors in Figs. 6 and 7, and correspondent phase difference

\begin{tabular}{llll}
\hline$H_{\mathrm{w}}(\mathrm{m})$ & $\begin{array}{l}\mathrm{HPA} \\
0.5 \rightarrow 3\end{array}$ & $\begin{array}{l}\text { OWSC } \\
0.5 \rightarrow 3\end{array}$ & $\begin{array}{l}\text { Difference } \\
0.5 \rightarrow 3\end{array}$ \\
\hline$\varphi_{\mathrm{FK}_{\mathrm{s}}}(\mathrm{deg})$ & $97 \rightarrow 117$ & $156 \rightarrow 136$ & $-59 \rightarrow-19$ \\
$\varphi_{\mathrm{FK}_{\mathrm{d}}}(\mathrm{deg})$ & $359 \rightarrow 355$ & $90 \rightarrow 98$ & $269 \rightarrow 257$ \\
$\varphi_{\mathrm{D}}(\mathrm{deg})$ & $167 \rightarrow 167$ & $80 \rightarrow 80$ & $87 \rightarrow 87$ \\
$\varphi_{\mathrm{R}}(\mathrm{deg})$ & $265 \rightarrow 287$ & $326 \rightarrow 307$ & $-62 \rightarrow-20$ \\
$\varphi_{\text {vis }}(\mathrm{deg})$ & $179 \rightarrow 176$ & $291 \rightarrow 309$ & $-112 \rightarrow-132$ \\
\hline
\end{tabular}

and velocity, respectively; in fact, since position and velocity are roughly $90^{\circ}$ out of phase, they rotate with a consistent phase difference, so static FK and radiation phasors rotate accordingly.

Finally, the viscous drag phasor rotation is quite small for the HPA and relatively large for the OWSC, consistent with the relative importance of the viscous drag term in each device. Note that, in the OWSC, the phasors of the two dissipative terms, namely radiation and viscous drag forces, are counter-rotating as the wave height increases. Furthermore, the phase difference between the radiation and viscous drag phasors decreases for larger wave amplitude; consequently, a more constructive interaction is achieved, making the total loss larger.

Comparing the phasor graphs of the HPA and the OWSC, in Figs. 6 and 7, respectively, it can be noted that the phasors of each hydrodynamic force component rotate in the opposite direction (the $\mathrm{FK}_{\mathrm{s}}$ phasor, for example, is rotating counter-clockwise for the HPA, but clockwise for the OWSC). Moreover, the range of phases of each phasor is different between the HPA and the OWSC (the HPA FK phasors, for example, are between $97^{\circ}$ and $117^{\circ}$, while the OWSC FK shasors are between $136^{\circ}$ and $156^{\circ}$ ). Table 1 tabulates the bounding phases for each force component, namely at the smallest and largest wave height, as well as the phase difference between the HPA and OWSC.

To have a more complete description of the relevance of nonlinearities in HPAs and OWSCs for all wave conditions considered in this paper, the FK ratio is defined as the ratio between the amplitude of FK forces/torques (static plus dynamic) and the total hydrodynamic force/torque. Likewise, the viscous drag ratio is defined as the ratio between the amplitude of the viscous drag force/torque, and the total hydrodynamic force/torque.

FK and viscous drag ratios are shown, respectively, in Figs. 8 and 9, where each line corresponds to a constant wave height. The HPA exhibits FK ratios from 0.81 to 0.96 , showing that FK forces constitute the largest part of the total hydrodynamic force. On the other hand, FK ratios for the OWSC are considerably smaller, ranging from 0.09 to 0.36 . Nevertheless, both the HPA and the OWSC show a similar

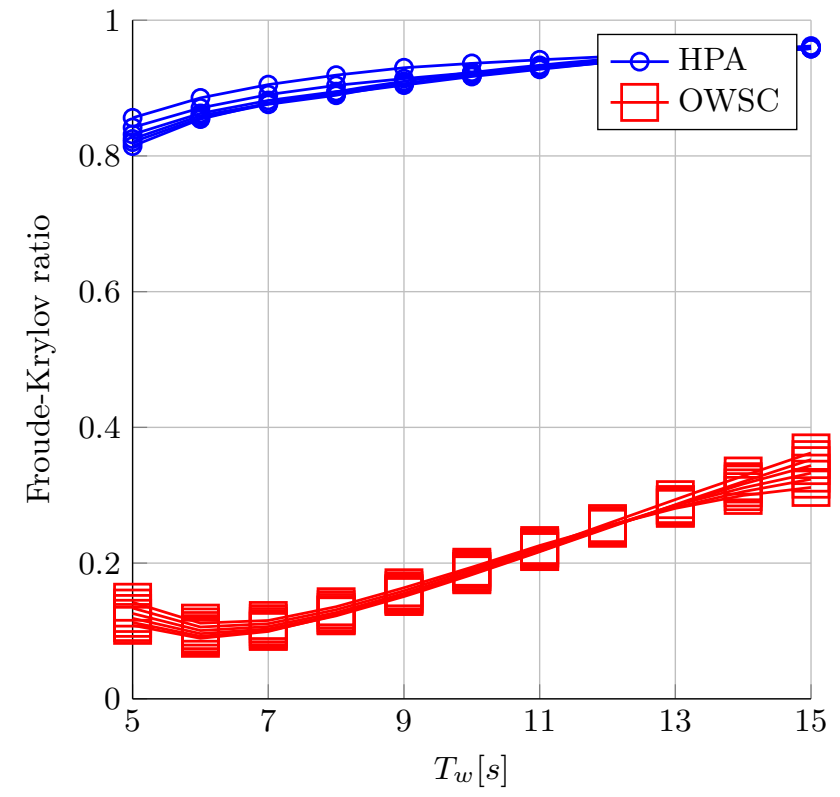

Fig. 8 Froude-Krylov force ratio, defined as the ratio between the FK force (static and dynamic) and the total hydrodynamic force. Each line corresponds to constant wave height, ranging from 0.5 to $3 \mathrm{~m}$ with a step of $0.5 \mathrm{~m}$

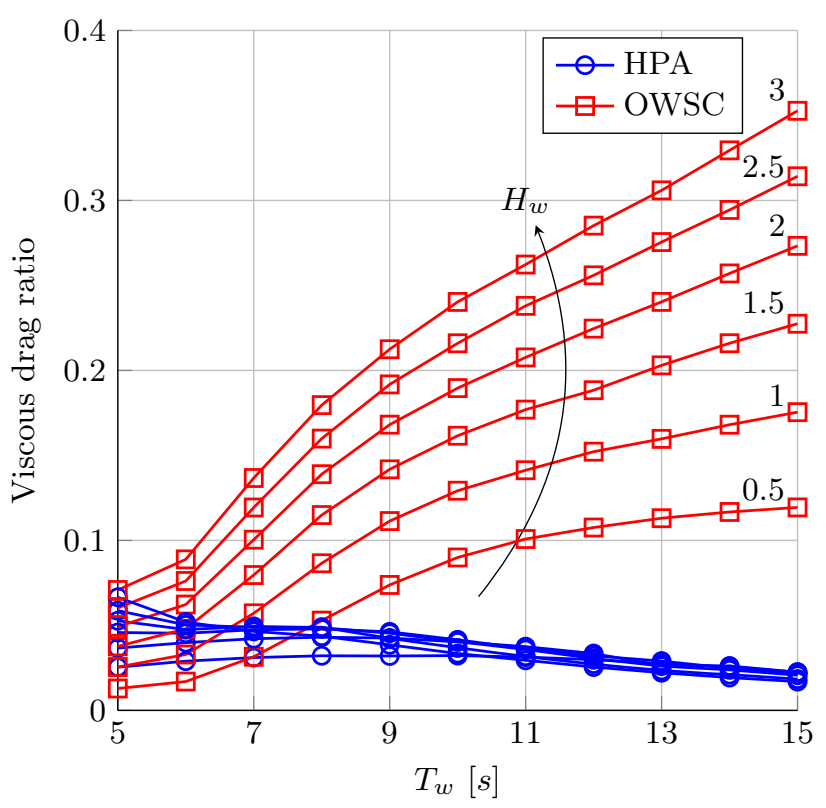

Fig. 9 Viscous drag ratio, defined as the ratio between the viscous drag force and the total hydrodynamic force. Each line corresponds to constant wave height (shown at the right hand side), ranging from 0.5 to $3 \mathrm{~m}$ with a step of $0.5 \mathrm{~m}$

trend: the FK ratio increases with the wave period $T_{\mathrm{w}}$, while it is independent of the wave height $H_{\mathrm{w}}$.

The viscous drag ratio in Fig. 9 shows a completely different trend, compared to Fig. 8. The viscous drag ratio of the HPA experiences little dependence on either wave period or height, especially at larger periods, and exhibits a general 


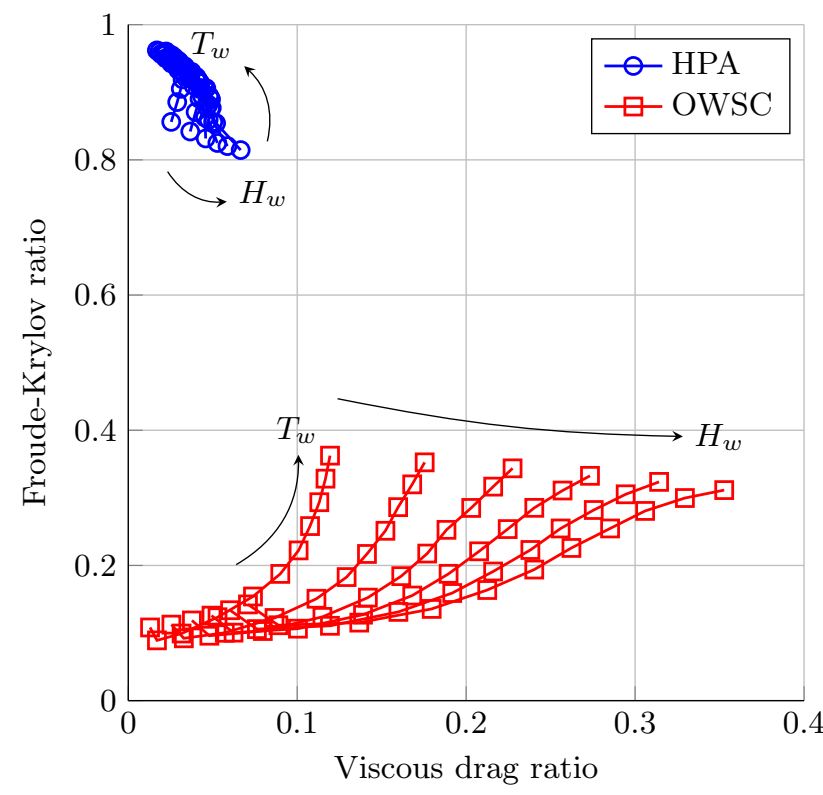

Fig. 10 Froude-Krylov ratio versus viscous drag ratio, defined as in Figs. 8 and 9, respectively. Every line has a constant wave height, ranging from 0.5 to $3 \mathrm{~m}$ with step $0.5 \mathrm{~m}$, increasing from left to right, with wave period $T_{\mathrm{w}}$, ranging from from 5 to $15 \mathrm{~s}$ with step $1 \mathrm{~s}$, increasing upwards along each line

slightly negative slope of the curves; since the FK forces are dominating the device dynamics, as shown in Fig. 8, the variations in viscous drag force have little impact on the overall hydrodynamic force, so the viscous drag ratio remains quite small (between 0.01 and 0.06). Furthermore, the negative slope suggests that the increase in viscous drag force is even smaller than the increase in FK force. On the other hand, the viscous drag ratio for the OWSC shows a strong dependence on both wave period and height. Moreover, the curves are clearly distinct because of the large values the viscous drag ratio assumes, highlighting its relevance with respect to the total hydrodynamic force.

It is useful to gather the information contained in Figs. 8 and 9 in a single compact graph, shown in Fig. 10, in order to highlight the essential differences between HPAs and OWSCs, and the relative importance of nonlinear FK and viscous drag effects. As shown by the arrows in Fig. 10, the cloud of points for each device presents different trends at constant wave heights $H_{\mathrm{w}}$, increasing from left to right, with the wave period $T_{\mathrm{w}}$ increasing upwards along each line.

The cloud of points for the HPA is compact and shows little dependence on different wave heights and periods, occupying a small area at the top left corner of the graph at high FK ratios (greater than 0.8), and low viscous drag ratios (smaller than 0.07).

On the contrary, a completely separate region of the graph in Fig. 10 is occupied by the OWSC, located at low FK ratios (lower than 0.4), and viscous drag ratios up to 0.35 . The
OWSC points are widely spread, showing a strong sensitivity to both wave period and height.

\section{Conclusions}

This paper deals with the major hydrodynamic differences existing between heaving point absorbers, and oscillating wave surge converters. The relative amplitude and phase of each hydrodynamic force component are investigated, using nonlinear models for Froude-Krylov and viscous drag forces. A particular focus is placed on the importance of nonlinearities; on the one hand, the sensitivity of scaling to hydrodynamic nonlinearity is examined, when a prototype scale model is used to predict the full-scale model performance; on the other hand, the relevance on nonlinear forces is discussed, with respect to the total hydrodynamic force.

From Fig. 5, it is clear that the mathematical structure of nonlinear Froude-Krylov and viscous drag forces is scale invariant. Therefore, eventual differences between experimental tests at prototype and full scales are not due to the inherent structure of the nonlinear forces, but may be attributed to different flow characteristics, which impact the model parameters (namely $C_{\mathrm{d}}$ ).

Nonlinearities also affect the phase with which the force components acting on the devices since, for a constant wave frequency, the phasors of the forces are rotating, as the the wave height increases.

Nonlinear forces show different relevance in HPAs and OWSCs. Viscous drag effects in HPAs are small compared to the other forces, while they are significant in OWSCs. The most important hydrodynamic force in HPAs is the nonlinear FK force, while diffraction and radiation are relatively small. Moreover, the dynamic FK force is the main wave excitation mechanism in HPAs, since the diffraction force is negligible. On the contrary, the dynamics of OWSCs are mainly driven by diffraction and radiation forces, while the dynamic FK force is relatively low.

Consequently, the relevance of nonlinear FK forces in HPAs appears to be considerably larger than the viscous drag force, since FK forces cover 81-96\% of the total hydrodynamic force. Such percentages remain consistent for different wave heights and periods. Conversely, the relative relevance of nonlinear FK forces and viscous drag in OWSCs is quite sensitive to wave parameters. Aside from the variability, FK forces are, in general, significantly more important in HPAs than in OWSCs, while viscous drag becomes much more relevant in OWSCs than HPAs.

Diffraction and radiation forces, which have been assumed to be linear, appear to be negligible in HPAs, while they are the most important hydrodynamic force components in OWSCs. A possible topic of future work would be to include a nonlinear representation of diffraction and radiation forces as 
well which, especially in OWSCs, could modify the balance between the different components of the total hydrodynamic force. A further possible topic of future work would be to completely validate the mathematical models used in this paper, for regular and irregular waves, using CFD simulations or experimental tests.

Acknowledgements This paper is based upon work supported by Science Foundation Ireland under Grant no. 13/IA/1886.

\section{References}

Aquamarine (2016) Aquamarine power. http://www.aquamarinepower. $\mathrm{com} /$

Babarit A, Hals J, Muliawan M, Kurniawan A, Moan T, Krokstad J (2012) Numerical benchmarking study of a selection of wave energy converters. Renew Energy 41:44-63

Bearman P, Downie M, Graham J, Obasaju E (1985) Forces on cylinders in viscous oscillatory flow at low keulegan-carpenter numbers. J Fluid Mech 154:337-356

Bhinder MA, Babarit A, Gentaz L, Ferrant P (2011) Assessment of viscous damping via $3 \mathrm{~d}$-cfd modelling of a floating wave energy device. In: Proceedings of the 9th European wave and tidal energy conference, Southampton

Bhinder MA, Babarit A, Gentaz L, Ferrant P (2012) Effect of viscous forces on the performance of a surging wave energy converter. In: Proceedings of the 22nd international and polar engineering conference, Rhodes, pp 545-549

Bhinder MA, Babarit A, Gentaz L, Ferrant P (2015) Potential time domain model with viscous correction and cfd analysis of a generic surging floating wave energy converter. Int J Mar Energy 10:7096. doi:10.1016/j.ijome.2015.01.005

Clabby D (2014) Wave energy conversion at prototype and model scales. $\mathrm{PhD}$ thesis, Queen's University Belfast

Clement A, Ferrant P (1988) Nonlinear water waves: IUTAM Symposium, Tokyo, August 25-28, 1987. In: Superharmonic waves generated by the large amplitude heaving motion of a submerged body. Springer, Berlin, pp 423-433

Cummins W (1962) The impulse response function and ship motion. Schiffstechnik 9:101-109

Falnes J (2002) Ocean waves and oscillating systems. Cambridge University Press, Cambridge

Folley M, Henry A, Whittaker T (2015) Contrasting the hydrodynamics of heaving and surging wave energy converters. In: Proceedings of the 11th European wave and tidal energy conference, Nantes
Gilloteaux JC (2007) Mouvements de grande amplitude d'un corps flottant en fluide parfait. Application à la récupération de l'énergie des vagues. $\mathrm{PhD}$ thesis, Ecole Centrale de Nantes-ECN

Giorgi G, Ringwood JV (2016) Computationally efficient nonlinear Froude-Krylov force calculations for heaving axisymmetric wave energy point absorbers. J Ocean Eng Mar Energy 3(1):21-33

Giorgi G, Ringwood JV (2017) Froude-krylov and viscous drag representations in nonlinear wave energy devices models in the computation/fidelity continuum. Ocean Eng 141:164-175

Giorgi G, Penalba M, Ringwood JV (2016a) Nonlinear hydrodynamic force relevance for different wave energy converter types. In: Proceedings of the $3 \mathrm{rd}$ asian wave and tidal energy conference, pp 154-162

Giorgi G, Penalba M, Ringwood JV (2016b) Nonlinear hydrodynamic models for heaving buoy wave energy converters. In: Proceedings of the 3rd asian wave and tidal energy conference, pp 144-153

Hansen RH (2013) Design and control of the powertake-off system for a wave energy converter with multiple absorbers. Videnbasen for Aalborg UniversitetVBN, Aalborg UniversitetAalborg University, Det Teknisk-Naturvidenskabelige Fakultet, The Faculty of Engineering and Science

Heller V (2011) Scale effects in physical hydraulic engineering models. J Hydraul Res 49(3):293-306

Keulegan GH, Carpenter LH (1956) Forces on cylinders and plates in an oscillating fluid. US Department of Commerce, National Bureau of Standards

Lok K, Stallard T, Stansby P, Jenkins N (2014) Optimisation of a clutchrectified power take off system for a heaving wave energy device in irregular waves with experimental comparison. Int J Mar Energy $8: 1-16$

Merigaud A, Gilloteaux JC, Ringwood JV (2012) A nonlinear extension for linear boundary element methods in wave energy device modelling. In: ASME 2012 31st international conference on ocean, offshore and arctic engineering, American Society of Mechanical Engineers, pp 615-621

Molin B (2002) Hydrodynamique des structures offshore. Editions Technip

Morison JR, Johnson JW, Schaaf SA (1950) The force exerted by surface waves on piles. J Pet Technol 2(5):149-154

Taghipour R, Perez T, Moan T (2007) Hybrid frequency-time domain models for dynamic response analysis of marine structures. Ocean Eng 35(7):685-705

Wavestar (2016) Wavestar a/s. http://wavestarenergy.com/

WAMIT Inc. (2013) WAMIT v7.0 user manual

Whittaker T, Collier D, Folley M, Osterried M, Henry A, Crowley M (2007) The development of oyster a shallow water surging wave energy converter. In: Proceedings of the 7th European wave and tidal energy conference, Porto, pp 11-14 